\title{
ACCIDENTALIDAD POR RIESGO BIOLÓGICO EN LOS ESTUDIANTES DE ENFERMERÍA DE LA UNIVERSIDAD DE CIENCIAS APLICADAS Y AMBIENTALES U.D.C.A, BOGOTÁ, COLOMBIA
}

\section{BIOHAZARD ACCIDENTALITY OF NURSING STUDENTS IN THE UNIVERSITY OF APPLIED AND ENVIROMENTAL SCIENCES U.D.C.A, BOGOTA, COLOMBIA}

\author{
Margarita María Orozco ${ }^{1}$ \\ ${ }^{1}$ Enfermera. Especialista en Gerencia de la Salud Ocupacional, Docente U.D.C.A, dirección para correspondencia: Calle 222 \\ No.55-37 Bogotá. D.C. Colombia. e-mail: morozco@udca.edu.co
}

Rev. U.D.C.A Act. \& Div. Cient. 16(1): 27 - 33, 2013

\section{RESUMEN}

La Enfermería es una disciplina enfocada al cuidado del paciente, la familia y la comunidad. Al ofrecer el cuidado, los estudiantes se exponen permanentemente a microrganismos biológicos causantes de enfermedades, debido a que su actividad implica el contacto directo con pacientes, potencialmente infecciosos. La probabilidad de un accidente por riesgo biológico en las prácticas clínicas es alta, por la falta de habilidad y de destreza al encontrarse en un proceso de formación profesional. El accidente expone al estudiante a adquirir enfermedades infecciosas importantes, como el Virus de la Inmunodeficiencia Humana (VIH), la Hepatitis B y C ( VHB, VHC), entre muchas otras, además del impacto psicosocial que genera en el alumno. Se realizó un estudio descriptivo, mediante la aplicación de una encuesta a 77 estudiantes, cuyo objetivo fue caracterizar los accidentes por riesgo biológico en las prácticas clínicas e identificar los conocimientos sobre accidentalidad en los alumnos de Enfermería de la Universidad de Ciencias Aplicadas y Ambientales U.D.C.A. Los resultados muestran un 15\% de accidentes en las prácticas asistenciales, el tipo de exposición más frecuente fueron las lesiones percutáneas o pinchazos con elementos cortopunzantes (40\%) y las salpicaduras (30\%), la actividad de enfermería más relacionada con la exposición fue la administración de medicamentos $(70 \%)$ y la causa principal del accidente, el estrés y la alta carga de actividades al ofrecer el cuidado de Enfermería (70\%). Se resalta la importancia del autocuidado y las medidas de bioseguridad, como herramienta fundamental frente a la prevención de la exposición biológica.
Palabras clave: Personal de enfermería, estudiantes de enfermería, riesgo biológico, accidente biológico.

\section{SUMMARY}

Nursing is a discipline focused on patient care, family and community. By providing the care, the students are permanently exposed to disease-causing biological microorganisms, since their activity involves direct contact with potentially infectious patients. The probability of an accident by biohazard in clinical practice is high due to their lack of skills and ability, being still in a training process. The accidents expose students to acquire important infectious diseases such as Human Immunodeficiency Virus (HIV), Hepatitis B and C (HBV, HCV), among many others, in addition to the psychosocial impact it has on the student. A descriptive study was performed by applying a survey to 77 students, with the goal to characterize the accidents with biological risk in clinical practice and identify the knowledge of possible accidents in nursing students at the University of Applied and Environmental Sciences U.D.C.A. The results showed a $15 \%$ of accidents in health care practices, being the most common type of exposure percutaneous injuries with sharps or needles (40\%) and splash (30\%). The nursing activity most related to exposure was medication administration (70\%) and the main cause of accidents, stress and high load of activities to provide nursing care $(70 \%)$. The importance of self-care and biosecurity measures as a fundamental approach to prevention of biological exposure.

Key-words: Nursing Staff, students, biohazard, biological accident and biosecurity measures. 


\section{INTRODUCCIÓN}

"El Riesgo Biológico se define como la exposición o contacto con sangre o fluidos corporales como orina, líquido amniótico, líquido pleural, entre otros que pueden estar contaminados con gérmenes infecciosos como bacterias, virus, hongos o parásitos", según Occupational Safety Health Administration OSHA (2003) y constituye uno de los riesgos más frecuentes y de mayor relevancia a nivel hospitalario. La exposición biológica puede generar infecciones causadas por diversos agentes, especialmente, virus y bacterias, que genera una amenaza permanente para el personal de la salud y, en especial, para los estudiantes de enfermería que, en su actividad diaria, deben manipular fluidos con riesgo biológico.

La exposición o accidente por riesgo biológico, se puede definir como una lesión percutánea producida por un pinchazo o un corte con un objeto afilado o corto punzante: de igual manera, el contacto con mucosas o piel no intacta (que presenta lesiones) con sangre, tejidos u otros líquidos corporales, que pueden ser potencialmente infecciosos y ponen en riesgo el personal de salud, para el contagio con enfermedades (CDC, 2001).

Se han detectado más de veinte enfermedades infectocontagiosas de origen biológico, que pueden ser adquiridas en el ambiente hospitalario; sin embargo, las principales enfermedades a las que se expone frecuentemente el personal de salud por el contacto con sangre o fluidos corporales de sus pacientes son: el Virus de Inmunodeficiencia Humana $(\mathrm{VIH})$, Hepatitis B (VHB), Hepatitis C (VHC). Según el CDC (2001), el riesgo de infectarse con el virus de la Hepatitis B (VHB) en un accidente laboral, a través de una aguja que contenga sangre contaminada es del 6 al 30\%; el riesgo de contagio con el virus de Hepatitis C (VHC) es del 3\% y para el virus de la Inmunodeficiencia Humana (VIH), del 0,3\%. Asimismo, en los contactos cutáneos mucosos a los ojos, nariz, o boca con sangre contaminada con $\mathrm{VIH}$, el riesgo es en promedio del $0,1 \%$.

De acuerdo con datos estimados de OSHA (2003), más de 5,6 millones de trabajadores del cuidado de salud y de seguridad pública en el mundo pueden estar potencialmente expuestos a estos tipos de virus.

Aproximadamente, cada año, ocurren entre 500.000 a 600.000 accidentes con riesgo biológico en Estados Unidos, Canadá y Alemania, individualmente. La frecuencia en nuestro país no se ha calculado para el personal de salud, ni para los estudiantes; solamente existen datos aislados en las distintas Instituciones Prestadoras de Servicios de Salud (IPS) y en las Administradoras de Riesgos Laborales (ARL).
Con el fin de prevenir este tipo de accidentes, el CDC (2001) implementó en 1987, con posteriores actualizaciones, la estrategia de las precauciones universales de bioseguridad y múltiples campañas educativas creadas para aumentar el conocimiento sobre sobre el riesgo biológico y prevenir la exposición del mismo.

El conocimiento de los mecanismos de exposición, los riesgos de transmisión y los métodos de prevención pueden ayudar a los trabajadores de la salud y al personal que labore en los ambientes hospitalarios, a crear un entorno laboral seguro (Tapias et al. 2010).

Las investigaciones demuestran que los estudiantes del área de la salud y, especialmente los de enfermería, no están libres del riesgo de sufrir un accidente biológico (Díaz \& Cadena, 2003). Además, la práctica en enfermería implica un alto riesgo de accidentes y la probabilidad de adquirir enfermedades infectocontagiosas es de alto impacto, porque sus prácticas clínicas les exige la necesidad de utilizar elementos cortantes o punzantes, el contacto permanente con pacientes potencialmente infectantes y la manipulación de sangre y fluidos corporales, en las actividades de brindar el cuidado de enfermería (Rodríguez et al. 2008; Rubio et al. 2008)

Además de lo anterior son factores que se aducen como explicación a la ocurrencia de accidentes, la inexperiencia y el escaso desarrollo de habilidades manuales, propias del ejercicio, al encontrarse en un proceso de construcción del conocimiento (Ortiz, 2003); asimismo, la percepción del riesgo a nivel individual y colectivo, la actitud hacia la bioseguridad y la falta de adherencia a la utilización de los elementos de protección personal (Herrera \& Gómez, 2003).

En Latinoamérica son escasas las investigaciones que refieren la accidentalidad biológica en los alumnos de enfermería; no obstante, estudios revisados a nivel nacional e internacional, en estudiantes de diferentes áreas de la salud, evidencian un alto porcentaje de accidentalidad biológica en los de enfermería, comparado con los demás del área de la salud (Fica et al. 2010; Franco et al. 2007). De igual manera, según se afirma, del 65 al $70 \%$ de los accidentes ocurren en el personal de enfermería (Soto \& Olano, 2004).

Los estudios, además de evidenciar una importante accidentalidad en los estudiantes, demuestran que el accidente por punción o por percutáneo con elementos cortopunzantes, es la primera causa de exposición, seguido del contacto directo y salpicaduras con sangre o con fluidos corporales (Ortiz, 2003; Romero et al. 2007).

De esta manera, las principales actividades de enfermería involucradas en la exposición biológica son la administración de medicamentos y la realización de procedimientos propios 
de enfermería. En relación a la evaluación del riesgo, evaluado como alto, en la mayoría de los estudios (Herrera \& Gómez, 2003).

Los estudiantes de la Facultad de Enfermería de la Universidad de Ciencias Aplicadas y Ambientales U.D.C.A, inician sus prácticas clínicas en el segundo semestre de la carrera y se ha identificado que podría existir un importante riesgo de accidentes de origen biológico por varios factores, como el contacto permanente con pacientes en turnos de 8 horas diarias, la falta de habilidad para realizar procedimientos propios de la profesión y la falta de adherencia a las medidas de bioseguridad.

La problemática es mucho más compleja, porque el Sistema de Riesgos Laborales de nuestro país al no considerarlos como fuerza laboral, los cobija mediante una póliza estudiantil, que solamente los cubre, de manera parcial, de las contingencias derivadas de un accidente de trabajo, pero no de una futura enfermedad laboral y sus consecuencias a nivel físico, psicológico y social. Problema abordado en otros estudios como el realizado por Herrera \& Gómez (2003).

La situación anterior pone al descubierto la magnitud del problema que representa para los estudiantes y para la Universidad, la posibilidad latente que estos adquieran una enfermedad grave, como resultado de un accidente biológico, sucedido durante la práctica clínica.

Por la problemática expuesta anteriormente sobre la exposición al riesgo biológico en los estudiantes de enfermería durante sus prácticas clínicas y sus graves consecuencias, además, los escasos estudios investigativos sobre el tema dirigido a la población objeto de estudio, se desarrolló esta investigación, cuyos objetivos fueron caracterizar la accidentalidad biológica e identificar los conocimientos sobre riesgo y accidente biológico en los estudiantes de la facultad de enfermería, con el fin de proponer medidas de prevención y control para mejorar la seguridad y el bienestar de los estudiantes.

\section{MATERIALES Y MÉTODOS}

Estudio descriptivo, de corte transversal, que caracterizó la accidentalidad por riesgo biológico en las diferentes prácticas de enfermería: cuidado básico, materno infantil, niño y adolescente, adulto y anciano, salud ocupacional, salud mental y psiquiatría y programas especiales, de los de estudiantes de segundo a noveno semestre de la Facultad Enfermería de la Universidad de Ciencias Aplicadas y Ambientales U.D.C.A, en el periodo comprendido entre el primer semestre de 2007 y el primer semestre de 2011, en Bogotá, D.C.

La población estuvo constituida por 308 estudiantes; como criterios de inclusión, se tuvo en cuenta que se encontraran matriculados de segundo a noveno semestre, inscritos en el primer periodo de 2011 y que hubieran realizado prácticas clínicas durante un período mínimo de un curso académico. Se excluyeron los estudiantes de enfermería de primer semestre, porque no habían realizado prácticas clínicas. La muestra seleccionada fue de 77 estudiantes, que se obtuvo a partir de un muestreo aleatorio simple y se calculó con una confiabilidad del $95 \%$ y un error del $10 \%$, con la siguiente formula:

$$
\mathrm{n}=\frac{N z^{2} \mathrm{pq}}{(N-1) e^{2}+z^{2} \mathrm{pq}}
$$

El instrumento aplicado fue una encuesta estructurada, voluntaria y anónima realizada por la autora y validada por pares académicos, la cual, contenía 16 preguntas, tipo selección múltiple: las variables utilizadas fueron edad, sexo, semestre académico, número de accidentes, notificación, esquema de vacunación, tipo de exposición, actividades realizadas en el momento de accidentarse, causas, conocimientos de bioseguridad, riesgo, accidente biológico, factores de riesgo biológico en las prácticas y protocolo postexposición, preguntas direccionadas a caracterizar la accidentalidad e identificar los conocimientos sobre riesgo y accidente biológico. Para la validación de la encuesta, se aplicó, previamente, una prueba piloto, a una submuestra de estudiantes de características similares a los encuestados; se hicieron modificaciones y ajustes a dos preguntas, hasta llegar al instrumento final.

La investigación tuvo como referencia la Resolución No 8430 de 1993, por la que se establecen las normas científicas, técnicas y administrativas para la investigación en salud. Fue considerada de riesgo bajo, porque no representó ningún riesgo para la población del estudio; de igual manera, se prevaleció el respeto a su dignidad, la protección de sus derechos y su bienestar. Con anticipación a la realización de la entrevista, se les aplicó a los estudiantes un consentimiento informado por escrito y se les explicó verbal y claramente sobre los objetivos y las características de la investigación; además, se les garantizó la confidencialidad de toda la información recolectada.

Para el análisis, la información fue procesada mediante el programa SPSS (Científica Packet Social Statistic), versión 11.0 para Windows.

\section{RESULTADOS Y DISCUSIÓN}

En el estudio, el $100 \%$ de la población encuestada pertenecía al género femenino, situación esperada en una profesión predominantemente femenina; el $70 \%$ se encontraba en un rango de edad entre los 21 y los 26 años. En cuanto al se- 
mestre que cursaban, el $50 \%$ de la muestra estaban matriculados entre el segundo al quinto semestre y el $50 \%$, de sexto a noveno semestre.

Las Instituciones Prestadoras de Servicios de Salud IPS donde los estudiantes se hallaban realizando las prácticas fueron el Hospital San Blas, 50\%; el Hospital San Rafael de Facatativá, 30\% y el Hospital la Victoria, 20\%. La totalidad de la población encuestada contaba con EPS vigente al momento de aplicarse la encuesta.

El 15\% de los encuestados manifestó haber presentado un accidente de origen biológico en el transcurso de su formación profesional; de estos estudiantes, el $90 \%$ reportó un accidente y, el $10 \%$, dos o más. En cuanto al semestre, el $80 \%$ refirió declarar el accidente entre el segundo y el quinto semestre de la carrera y un $20 \%$, entre sexto y noveno semestre de la carrera. De igual manera, los semestres donde se reportaron más accidentes biológicos fueron el tercero y el quinto, donde se ven los cursos de cuidado de enfermería materno infantil y cuidado de enfermería al adulto y anciano.

El comportamiento anterior puede ser ocasionado por la falta de habilidad y de destreza de los estudiantes durante los primeros semestres de la carrera, en donde se realiza el aprendizaje de actividades propias del cuidado de enfermería, como la administración de medicamentos y la realización de procedimientos básicos.

Los encuestados refirieron la posibilidad de un importante subregistro por el desconocimiento del proceso de notificación, la baja percepción del riesgo o por temores de índole académico hacia los docentes, con quien realizan sus prácticas.

La información anterior es corroborada con resultados de otros estudios nacionales e internacionales, como Warley et al. (2009) y Saliba et al. (2009), quienes encontraron una importante incidencia de accidentes de origen biológico y un riesgo relativo mayor en los estudiantes de enfermería, respecto a otras carreras de la salud (Fica et al. 2010).

En cuanto al tipo de exposición y similar a otros estudios, como el de Fica et al. (2010) y Ortiz (2003), en el presente trabajo predominó la exposición percutánea, como los pinchazos, con un $40 \%$, las salpicaduras a piel intacta, con un $30 \%$ y el contacto directo de sangre o fluidos corporales con piel no intacta, en un $30 \%$.

Es importante destacar que la exposición percutánea es la más frecuente en enfermería, por la manipulación permanente de elementos cortopunzantes en las actividades y en los procedimientos propios de la profesion (Saliba et al. 2009). De igual manera, un porcentaje alto de accidentes por salpicadura puede indicar dificultades, bien sea por falta de capacitación o hábitos para incorporar las medidas de precaución universal, en las actividades que realizan los estudiantes en sus prácticas (Fica et al. 2010).

Entre las actividades que realizaban en el momento del accidente, el $70 \%$ respondió las relacionadas con la administración de medicamentos (incluye la preparación y la administración), un $20 \%$ refirió procedimientos propios de enfermería, como la curación de heridas y un $10 \%$, otra actividad diferente. La información obtenida demuestra que la administración de medicamentos, se constituye en la principal actividad relacionada con la accidentalidad en enfermería y coincide con el estudio de Romero et al. (2007).

Al respecto de las causas de ocurrencia de los accidentes, el $70 \%$ de los estudiantes refirieron, en primer lugar, el estrés y la alta carga de trabajo o actividades; en segunda instancia, con un $20 \%$, la falta de uso de elementos de protección personal y, por último, con un $10 \%$, por descuido propio, resultados que coinciden con estudios, como el de Warley et al. (2009), quienes encontraron en su investigación que la sobrecarga de trabajo y la carencia de elementos de protección personal son las principales causas para no cumplir con las medidas de precaución universal y la posibilidad de accidentarse.

De igual manera, Tapias (2010) en su estudio, además del estrés por la alta demanda de trabajo, refiere otras condiciones no menos importantes, como la falta de concentración, la fatiga por horarios extendidos, la falta de sueño y la carga emocional por la muerte de los pacientes.

En lo concerniente a la falta de adherencia de los estudiantes a la utilización de los elementos de protección personal, se evidencia la necesidad de sensibilización y capacitación frente a las medidas de precaución universal, como herramienta indispensable para la prevención de accidentes biológicos.

Al analizar el riesgo de adquirir infecciones transmitidas por líquidos corporales, según el protocolo del CDC (2001), se registró que un $40 \%$ de los accidentes, se clasifican en riesgo alto (accidentes percutáneos o pinchazo), un 30\%, riesgo medio (contacto directo a piel no intacta) y un $30 \%$, riesgo bajo (salpicaduras contacto directo a piel intacta). Esta situación evidencia la problemática de este grupo poblacional y la vulnerabilidad para adquirir enfermedades de alto impacto, como el HIV y Hepatitis B, por la posibilidad de seroconversión. Cabe resaltar que el $90 \%$ de los estudiantes refirieron tener el esquema de vacunación completo.

En relación a la actuación postexposicion, el $90 \%$ de los estudiantes respondieron que notificaron el accidente inmediatamente y lo hicieron a la docente de práctica. Estos datos son 
sensiblemente superiores a los evidenciados por Ortiz (2003), quien en su estudio encontró que solamente un $34,5 \%$ de los estudiantes realizaron el reporte oportunamente.

En cuanto a los conocimientos de los estudiantes sobre riesgo biológico y accidentalidad, se evidenció que el $68 \%$ de los estudiantes conocen la definición correcta de riesgo biológico y el $52 \%$, la definición de accidente biológico; asimismo, el $93 \%$ conoce los factores de riesgo biológico, a los cuales, se encuentra expuesto en sus prácticas.

Al indagarse sobre la claridad y el conocimiento de los protocolos de actuación, en caso de un accidente en las prácticas clínicas, el $80 \%$ respondió afirmativamente, comportamiento inferior al relacionado en otros estudios, como el de Guzmán et al. (2011).

En relación a los conocimientos sobre bioseguridad, se registró que un $30 \%$ de los estudiantes que se han accidentado conoce la definición correcta de bioseguridad y un $60 \%$ tiene conocimiento adecuado sobre las precauciones universales. Los anteriores resultados son similares a los presentados por Casanova et al. (2007).

De igual manera, en cuanto a los conocimientos que tienen los estudiantes sobre la acción prioritaria en caso de un accidente percutáneo por pinchazo, según lo recomendado por el CDC (2001), un 50\% respondió correctamente lavar el sitio de punción exhaustivamente con abundante agua y jabón; referente a la pregunta sobre la acción prioritaria ante un accidente por salpicadura, el $75 \%$ respondió acertadamente: lavado exhaustivo con agua y jabón, en salpicaduras cutáneas y lavado con solución salina, en salpicaduras a mucosas.

Se puede resaltar que la falta de conocimientos puede ser una de las causas de accidentalidad, ya que un mejor conocimiento de las variables que convergen en el accidente, podría fomentar la prevención de la exposición.

Se concluye que la accidentalidad biológica en los estudiantes de enfermería de la U.D.C.A es similar a la de otros estudios. El riesgo que corren es alto, si se tiene en cuenta la probabilidad de adquirir enfermedades graves y la falta de cobertura en materia de riesgos laborales en nuestro país. Se resalta, como aspecto positivo, una importante notificación de los mismos.

La exposición percutánea es la más frecuente en los estudiantes, por la utilización de elementos cortopunzantes en la actividad de preparar y de administrar medicamentos, por este motivo es necesario formar a los estudiantes en la realización de prácticas seguras, para prevenir incidentes y accidentes de esta naturaleza.
La mayor accidentalidad en los estudiantes de los primeros semestres refleja la falta de habilidad y de destreza al realizar los procedimientos propios de la profesión y la falta de conocimientos en torno al riesgo biológico y los protocolos postexposición.

Aun, cuando en cada semestre de la carrera se aborda el tema de bioseguridad, los resultados obtenidos evidencian falta de conocimientos sobre el riesgo biológico y los protocolos pre y post exposición, por este motivo, se recomienda incluir estas temáticas, que son desarrolladas ampliamente cuando cursan séptimo semestre, en la asignatura de cuidado de enfermería en salud ocupacional, desde los primeros semestres de la carrera.

El comportamiento de la accidentalidad biológica en los estudiantes de enfermería refleja que no son incidentes fortuitos, puesto que concurren factores controlables y evitables, que aumentan su riesgo de aparición. Por lo anterior, se recomienda a la Universidad y concretamente a la Facultad de Enfermería, tomar medidas inmediatas, que permitan formar a los estudiantes desde el primer semestre en la cultura de prevención del riesgo ocupacional.

De igual manera, como lo afirman Díaz \& Cadena (2003), desde la perspectiva académica es responsabilidad de la Universidad velar porque las condiciones y los ambientes donde se desarrollan las prácticas clínicas sean espacios seguros para los estudiantes. Por esta razón, se debe involucrar diferentes actores, como las instituciones hospitalarias, para que participen activamente en el desarrollo e implementación de estrategias, que permitan minimizar la exposición biológica, tanto en los estudiantes como en sus funcionarios $\mathrm{y}$, a la Universidad, para que se busque concientizar a los estudiantes frente a las consecuencias del riesgo biológico, mediante estrategias de prevención primaria o de prevención de la exposición.

Las estrategias pueden incluir, en primer lugar, el cumplimiento estricto de las medidas de precaución universal, la identificación de los riesgos o peligros, la capacitación en todos los aspectos que involucran la accidentalidad, la vacunación pre y post exposición y el análisis de investigación de los accidentes, para tomar medidas preventivas y correctivas, que mejoren la seguridad y el bienestar de los alumnos.

Por último, se recomienda realizar estudios de esta naturaleza, que aborden el personal de salud y, específicamente, el gremio de enfermería, con el fin de sensibilizar a la población y a las instituciones gubernamentales responsables de los riesgos laborales, en la implementación de estrategias y el desarrollo de políticas para la prevención del riesgo biológico y facilitar los entornos laborales saludables. 
Financiación: El presente estudio fue financiado por la Universidad de Ciencias Aplicadas y Ambientales U.D.C.A. Conflicto de intereses: Esta investigación y el manuscrito fue preparado y revisado por la autora, quien declara que no existe ningún conflicto de intereses que ponga en riesgo la validez de la presente publicación.

\section{BIBLIOGRAFÍA}

1. CASANOVA, S.; SANTOLARIA, E.; FORCADA, J.A.; BUEDO, V.E. 2007. Prevención del riesgo biológico en profesionales Sanitarios de la comunidad Valenciana. Edita: Generalitat. Conselleria de Sanitat, (España) $1^{\text {a }}$ ed. p. 34 y 35. Disponible desde Internet en: http://publicaciones.san.gva.es/publicaciones/documentos/V.4176-2007.pdf (con acceso 28/06/10)

2. CENTERS FOR DISEASE CONTROL AND PREVENTION -CDC-. 2001. Updated US Public Health Service guidelines for the management of occupational exposures to HBV, HCV, and HIV and recommendations for postexposure prophylaxis. MMWR 2001; 50(No. RR-11):1-42 Disponible desde Internet en: http://www.cdc.gov/mmwr/preview/mmwrhtml/ rr5011a1.htm (con acceso 30/06/10)

3. DÍAZ, L.A.; CADENA, L.P. 2003. Riesgo de hepatitis B entre estudiantes de medicina peruanos, luego de exposición a sangre y líquidos corporales. Rev. Gastroenterol. (Perú). 23(2):107-110.

4. FICA, C.; JEMENAO, P.; RUIZ, G.; LARRONDO, M.; HURTADO, C.; MUÑOZ, G.; SEPULVEDA, C. 2010. Accidentes por riesgo biológico entre estudiantes de carreras de la salud. Cinco años de experiencia. (Chile) Rev. Chil. Infectol. 27(1):34-39.

5. FRANCO, J.; MARÍN, A.; OCAMPO, L.M.; QUIROZ, T.; DÍAZ, P.A. 2007. Factores laborales y personales frente a la ocurrencia de accidentes de trabajo biológicos en el personal de enfermería de la clínica Villapilar ESE Rita Arango Álvarez del Pino Manizales (Caldas). Rev. Hacia Prom. Salud. (Colombia) (12):133-144. Disponible desde Internet en: http:// promocionsalud.ucaldas.edu.co/downloads/Revista\%2012_11.pdf (con acceso 28/06/10).

6. GUZMÁN, M.I.; EXPOSITO, R.; ARMENTEROS, E.; ALMAGRO, M.; GUZMÁN, L.; MARQUES, M.T. 2011. Accidentes biológicos por exposición percutánea en estudiantes de enfermería de la Universidad de Jaén, durante sus prácticas clínicas. Rev. Paraninfo digital. (España) 11:1-16. Disponible desde Internet en: http://www.index-f.com/para/n11-12/050d.php (con acceso 10/04/10).

7. HERRERA, A.; GÓMEZ, R. 2003. Accidentes por riesgos biológicos en estudiantes de la salud la Universidad Tecnológica de Pereira. Rev. Med. Risaralda. (Colombia). 9(1). Disponible desde Internet en: http://www. utp.edu.co/facies/revmedica/vol9n1/articulo3.htm (con acceso 10/04/10)

8. ORTIZ, S. 2003. Riesgos biológicos de los estudiantes de enfermería. Enfermería Clínica. (13):285-289 Disponible desde Internet en: http://dialnet.unirioja.es/servlet/articulo?codigo $=747522$ (con acceso $10 / 06 / 10)$

9. OSHA (Occupational safety and Health Administration). 2003. OSHA 3134. Exposición a Patógenos Transmitidos por la Sangre en el Trabajo. Departamento del trabajo EE.UU. Seguridad y Salud Ocupacional. Disponible desde Internet en: http://www.osha-slc. gov/Publications/OSHA3134/osha3134.html (con acceso 10/04/10)

10. RODRÍGUEZ, M.; PEREZ, M.; PLA, J.; MIRANDA, L.; GAROTTE, M.; PEÑA, M.; VALDEZ, M. 2008. Riesgos biológicos laborales en el personal de enfermería de una institución hospitalaria, ciudad de La Habana, septiembre 2006-abril 2007. (Cuba). 8(2):e2722. Disponible desde Internet en: http:// www.mednet.cl/link.cgi/Medwave/Enfermeria/2006/ marzo2008/2722 (con acceso 28/08/11).

11. ROMERO, A.; CABRERA, F.; JIMENEZ, M.; MUNOZ, J.C.; AVILA, I. 2007. Prevalencia de accidentes biológicos por punción accidental en el Hospital Universitario Virgen de la Victoria. Rev. Nure Invest. 31: 1-10. Disponible desde Internet en: http://www.nureinvestigacion.es/FICHEROS ADMINISTRADOR/ PONENCIA/pdf_comunicacion_12792007105419. pdf (con acceso 10/05/10)

12. RUBIO, M.; AVILA, G.; ARANA, B. 2008. Actitudes de Estudiantes de Enfermería mexicanos al manejar residuos peligrosos biológicos infecciosos. (México) Esc. Anna Nery, 2008, 12(3):479-484. Disponible desde Internet en www.scielo.br/pdf/ean/v12n3/ v12n3a13.pdf (con acceso 10/05/11)

13. SALIBA, C.; MARTINS, R.; ISPER, A.; da COSTA, L. 2009. Conductas de los Estudiantes del area de la salud frente a la exposición ocupacional a sangre y fluidos biologicos. Rev. Cienc. Trab. 11(31):18-21. Disponible desde Internet en: http://dialnet.unirioja. 
es/servlet/articulo?codigo $=3219089$ (con acceso $10 / 08 / 10)$

14. SOTO, V.; OLANO, E. 2004. Conocimiento y cumplimiento de medidas de bioseguridad en personal de enfermería. Hospital Nacional Almanzor Aguinaga. Chiclayo 2002. (Perú). Anales Fac. Med. 65:103-110.

15. TAPIAS, L.F.; TORRES, S.; TAPIAS, L.; SANTAMARIA, C.L. 2010. Accidentes biológicos en médicos residentes de Bucaramanga, Colombia. Rev. Col. Cir. 25(4):290-299. Disponible en internet: < http://www. scielo.org.co/scielo.php. (con acceso 10/08/10)
16. WARLEY, E.; PEREYRA, N.; DESSE, J.; CETANI, S.; de LUCA, A.; TAMAYO, N.; SZYLD, E. 2009. Estudio sobre la exposición ocupacional a sangre y fluidos corporales en el personal de Enfermería de un hospital de referencia de Buenos Aires, Argentina. Rev. Panam. Salud Pública. 25(6):524-529.

Recibido: Diciembre 13 de 2012

Aceptado: Abril 17 de 2013 